Copyright $@ 2010$ Institute of Electrical and electronics Engineers, Inc.

All Rights reserved.

Personal use of this material, including one hard copy reproduction, is permitted.

Permission to reprint, republish and/or distribute this material in whole or in part for any other purposes must be obtained from the IEEE.

For information on obtaining permission, send an e-mail message to stds-igr@ieee.org.

By choosing to view this document, you agree to all provisions of the copyright laws protecting it.

Individual documents posted on this site may carry slightly different copyright restrictions.

For specific document information, check the copyright notice at the beginning of each document. 


\title{
Discrete-Time Analogs for a Class of Continuous-Time Recurrent Neural Networks
}

\author{
Pingzhou Liu and Qing-Long Han
}

\begin{abstract}
This paper is concerned with the problem of local and global asymptotic stability for a class of discrete-time recurrent neural networks, which provide discrete-time analogs to their continuous-time counterparts, i.e., continuous-time recurrent neural networks with distributed delay. Some stability criteria, which include some existing results as their special cases, are derived. A discussion about the dynamical consistence of discrete-time neural networks versus their continuous-time counterparts is provided. An unconventional finite difference method is proposed and an example is also given to show the effectiveness of the method.
\end{abstract}

Index Terms-Delays, discrete-time analogs, recurrent neural networks, stability.

\section{INTRODUCTION}

$\mathbf{I}$ $\mathrm{N}$ standard neural networks theory, neural activity is described in terms of rates. The rate $v_{i}$ of neural $i$ is an analog variable which nonlinearly depends upon the excitation $x_{i}$ of the neuron

$$
v_{i}=g_{i}\left(x_{i}\right)
$$

where $g_{i}(\cdot)$ is usually taken as a sigmoid function. The excitation is given by a linear sum over input connections

$$
x_{i}=\sum_{i \in \Gamma_{i}} w_{i j} v_{j}
$$

where $v_{j}$ is the output rate of a presynaptic neuron $j$. The sum runs over all neurons which send signal to neuron $i$. The parameter $w_{i j}$, called synaptic efficacy, is the weight attributed to the connection from $j$ to $i$.

Equations (1) and (2) can be summarized in a single equation

$$
v_{i}=g_{i}\left(\sum_{j \in \Gamma_{i}} w_{i j} v_{j}\right) \text {. }
$$

The expression (3) is a static equation. It applies to the situation where a stationary input (a set of firing rates $v_{j}$ ) is mapped to a stationary output (the rate $v_{i}$ ).

Manuscript received June 15, 2006; revised December 19, 2006; accepted December 26, 2006. This work was supported by the Informatics and Communication Faculty's Advanced Research Grants Scheme Project entitled "Discrete-time analogs of recurrent neural networks," (Oct. 2004-Dec. 2004) and by the Central Queensland University for the Research Advancement Awards Scheme Project entitled "Robust Fault Detection, Filtering and Control for Uncertain Systems with Time-Varying Delay" (Jan. 2006-Dec. 2008).

P. Liu is with the School of Computing Sciences, Faculty of Business and Informatics, Central Queensland University, Rockhampton, Qld 4702, Australia, on leave from Flinders University, South Australia and Shanxi Normal University, China.

Q.-L. Han is with the School of Computing Sciences, Faculty of Business and Informatics, Central Queensland University, Rockhampton, Qld. 4702, Australia (e-mail: q.han@cqu.edu.au).

Digital Object Identifier 10.1109/TNN.2007.891593
In order to make the equation time-dependent, a straightforward way to introduce dynamics into the rate (3) is to replace it by a continuous-time differential equation [1], [8], [9], [21]

$$
\tau_{i} \frac{d x_{i}(t)}{d t}+x_{i}(t)=\sum_{j=1}^{m} w_{i j} g_{j}\left(x_{j}(t)\right), \quad i=1,2, \ldots, m \text {. }
$$

The system (4) is usually called the Hopfield neural network [9]. It has been customary to impose several types of restrictions on the elements of the interconnection matrix and the activity functions to guarantee the stationary state (2) or (3) of the neural network (4) to be a global attractor.

One limitation of model (4) is that it ignores interneuron propagation delays. But, neural networks usually have a spatial extent due to the presence of a multitude of parallel pathways with a variety of axon sizes and lengths. Thus, there is a distribution of conduction velocities along these pathways and a distribution of propagation delays. In these circumstances, the signal propagation is not instantaneous. The value of the state variable in the past, which affects its present dynamics, is determined by a synaptic filter or a delay kernel. Therefore, one may express the synapse input to the activity function as the following convolution integral [19]:

$$
k_{i j}(t) * g_{j}\left(x_{j}(t)\right)=\int_{\varsigma}^{t} k_{i j}(t-s) g_{j}\left(x_{j}(s)\right) d s
$$

where $\varsigma=-\infty$ or $\varsigma=0$. Typically, each synaptic filter is assumed as causal, i.e., $k_{i j}(t)=0$, while $t<0$. Consequently, if $\varsigma=-\infty$, we have the system

$$
\begin{array}{r}
\tau_{i} \frac{d x_{i}}{d t}+x_{i}=\sum_{j=1}^{m} w_{i j} \int_{-\infty}^{t} k_{i j}(t-s) g_{j}\left(x_{j}(s)\right) d s \\
i=1,2, \ldots, m
\end{array}
$$

where the synaptic filter has infinite memory; if $\varsigma=0$, the synaptic filter has finite memory and then, we have

$$
\begin{array}{r}
\tau_{i} \frac{d x_{i}}{d t}+x_{i}=\sum_{j=1}^{m} w_{i j} \int_{0}^{t} k_{i j}(t-s) g_{j}\left(x_{j}(s)\right) d s, \\
\quad i=1,2, \ldots, m .
\end{array}
$$

System (6) is well known as a recurrent neural network model with distributed delay [5], [6], [16], [19], [29]. But for system (7), to the best of our knowledge, little research has focused on it. The initial condition of system (7) can be given as

$$
x_{i}(t)=\phi_{i}(t), \quad 0 \leq t \leq t_{0}, \quad i=1,2, \ldots, m .
$$

If $t_{0}=0$, the function $\phi_{i}$ reduces to an initial vector $\phi(0)=x_{0}$. In practical situations, a system always starts at time $t=0$. If the system has been switched off and restarts at time $t=t_{0}$, the memory from 0 to $t_{0}$ may influence the system's operation. 
In the case of infinite memory of system (6), the assumption of memory of all the past is a simplification. In fact, through a simple transformation, system (6) can be rewritten as

$$
\begin{array}{r}
\tau_{i} \frac{d x_{i}}{d t}+x_{i}=\sum_{j=1}^{m} w_{i j} \int_{0}^{\infty} k_{i j}(s) g_{j}\left(x_{j}(t-s)\right) d s \\
i=1,2, \ldots, m
\end{array}
$$

which is autonomous from the view of dynamical systems.

System (7) is a typical Volterra integrodifferential equation. Recently, Liu and Han [19] have studied system (7) and its relationship with system (6). The results in [19] have unified many existing results in the literature about recurrent neural networks with or without delays.

For continuous-time recurrent neural networks, there are many results available in the literature. For example, recurrent neural networks have successfully been applied to solving nonlinear convex programs, subject to linear constraints and convex quadratic programs [20], [26], [27], while projecting neural networks for addressing constrained optimization problems [11], [23]-[25], [28]. However, in sequence processing, we use discrete-time iteration rather than continuous-time as neurons may have no temporal response. In the cases of designing computer simulations or sampling, without additional conditions or information about discrete or sampling time intervals, one would not expect that the networks can be completely represented by their discrete counterparts or recoverable from the samples. Some methods for analyzing discrete-time systems and their continuous-time counterparts may look similarly, but the analysis of continuous-time networks may not be applicable to their discrete-time version. Therefore, the detailed analysis for discrete-time models is necessary and important. Recently, a discrete-time recurrent neural network, which is a discrete-time analog of system (4), has been studied and some criteria for global stability of the equilibrium have been given in [10], [12], and [13].

In this paper, first, we will model discrete-time analogs of systems (6) and (7). These discrete-time analogs can be regarded as stand alone discrete-time recurrent neural networks with discrete-time distributed delay. Second, we will study their local and global stability. Third, we will discuss the complexity of discrete-time recurrent neural networks versus their continuoustime counterparts and propose an unconventional finite difference method for discretizing continuous-time recurrent neural networks. Finally, we will give some concluding remarks of the results.

\section{Discrete-Time RECURRENT NEURAL NETWORKS}

For sequence processing using a neural network, we may introduce dynamics into the rate (3) by the following difference system:

$$
\tau_{i} \triangle x_{i}[n]+x_{i}[n]=\sum_{j=1}^{m} w_{i j} g_{i}\left(x_{j}[n]\right)
$$

where $\triangle x_{i}[n]=x_{i}[n+1]-x_{i}[n]$. To distinguish between continuous-time and discrete-time processes, we use $n$ to denote the discrete-time independent variable and brackets [-] to enclose $n$.
The sampling operation also generates a discrete-time signal from a continuous-time signal. In this case, we may define a discrete-time signal $x_{i}[n]$ that is equal to the samples of $x_{i}(t)$ at integer multiples of a sampling interval $T$, that is $x_{i}[n] \triangleq$ $x_{i}(n T)$.

For computer simulation of a continuous-time system, one can use finite difference algorithm to generate a discrete-time system. For example, the forward Euler's approximation of system (4) with discrete step $h$ will lead to

$$
x_{i}[n+1]=a_{i} x_{i}[n]+\sum_{j=1}^{m} b_{i j} g_{j}\left(x_{j}[n]\right)
$$

where $a_{i} \triangleq\left(1-h / \tau_{i}\right), b_{i j} \triangleq\left(h / \tau_{i}\right) w_{i j}$, and $x_{i}[n] \triangleq x_{i}(n h)$. The previous first-order nonlinear difference system (11) has been studied in [10], [12], and [13].

In the following, we consider a recurrent neural network with discrete-time distributed delay described by a discrete-time scale nonlinear system of the form:

$$
\begin{array}{r}
x_{i}[n+1]=a_{i} x_{i}[n]+\sum_{j=1}^{m} b_{i j} \sum_{\nu=-\infty}^{n} k_{i j}[n-\nu] g_{j}\left(x_{j}[\nu]\right), \\
i=1,2, \ldots, m
\end{array}
$$

for infinite memory or

$$
\begin{array}{r}
x_{i}[n+1]=a_{i} x_{i}[n]+\sum_{j=1}^{m} b_{i j} \sum_{\nu=0}^{n} k_{i j}[n-\nu] g_{j}\left(x_{j}[\nu]\right), \\
i=1,2, \ldots, m
\end{array}
$$

for finite memory.

The inside sum of the systems (12) and (13), i.e.,

$$
\sum_{\nu=\varsigma}^{n} k_{i j}[n-\nu] g_{j}\left(x_{j}[\nu]\right)
$$

where $\varsigma=-\infty$ or $\varsigma=0$, is the so-called convolution sum. If one chooses the delayed kernels $k_{i j}(\cdot)$ as the discrete $\delta$-function

$$
\delta[n]= \begin{cases}1, & n=0 \\ 0, & \text { otherwise }\end{cases}
$$

then systems (12) and (13) become system (11).

In general, by choosing appropriate delayed kernels, systems (12) and (13) include all higher order difference systems that can be considered as discrete analogs of any infinite and finite delayed neural networks models. However, systems (12) and (13) are not usual difference systems; they are so called nonlinear Volterra difference systems. Thus, the standard theory about difference systems would not work on them.

Systems (12) and (13) may be represented by the following compact vector forms:

$$
\mathbf{x}[n+1]=A \mathbf{x}[n]+B \sum_{\nu=-\infty}^{n} K[n-\nu] G(\mathbf{x}[\nu])
$$


and

$$
\mathbf{x}[n+1]=A \mathbf{x}[n]+B \sum_{\nu=0}^{n} K[n-\nu] G(\mathbf{x}[\nu])
$$

respectively, where $\mathbf{x}=\left[x_{1}, x_{2}, \ldots, x_{m}\right]^{T}$ is the state vector of the network, $A=\operatorname{diag}\left[a_{1}, a_{2}, \ldots, a_{m}\right], B=\left[b_{i j}\right]_{m \times m}$ is the real-valued matrix of the synaptic connection weights, $K(\cdot)=\left[k_{i j}(\cdot)\right]_{m \times m}$ is the delayed kernels matrix, and $G(x)=$ $\left[g_{1}\left(x_{1}\right), g_{2}\left(x_{2}\right), \ldots, g_{m}\left(x_{m}\right)\right]^{T}$ is a vector of nonlinear activation function.

In this paper, we focus on the stability analysis for a class of discrete-time recurrent neural networks, where activation functions are globally Lipschitz continuous of classes $\mathcal{L}, \mathcal{G L}$, or $\mathcal{S}$. If $G(\cdot) \in \mathcal{L}$, then there exist $\ell_{i}$ such that $\forall \theta, \rho \in R$, and $\theta \neq \rho$

$$
\left|g_{i}(\theta)-g_{i}(\rho)\right| \leq \ell|\theta-\rho| .
$$

If $G(\cdot)$ is also monotone nondecreasing, i.e., $g_{i}(\cdot) \in \mathcal{G L}$, then there exist $\underline{\ell}_{i}$ and $\bar{\ell}_{i}$ such that $\forall \theta, \rho \in R$, and $\theta \neq \rho$

$$
0 \leq \underline{\ell}_{i} \leq \frac{g_{i}(\theta)-g_{i}(\rho)}{\theta-\rho} \leq \bar{\ell}_{i}
$$

In addition, if $G(\cdot)$ is also differentiable, i.e., $G(\cdot) \in \mathcal{S}$, then

1) $g_{i} \in C^{1}(R)$ and $g_{i}^{\prime}(x)>0$ for $x \in R$, and $g_{i}^{\prime}(0)=$ $\sup _{x \in R} g_{i}^{\prime}(x)>0$

2) $g_{i}(0)=0$ and $g_{i}^{\prime}(x) \rightarrow \pm 1$, as $x \rightarrow \pm \infty$.

Obviously, $\mathcal{S} \subset \mathcal{G} \mathcal{L} \subset \mathcal{L}$.

In order to analyze the properties of systems (12) and (13), in the remainder of this section, we will give some notations and some preliminary results about linear Volterra difference systems.

Let $R^{m}$ denote a real $m$-dimensional Euclidean space of column vectors $\mathbf{x}=\operatorname{col}\left(x_{1}, x_{2}, \ldots, x_{m}\right)$ with the Euclidean norm $\|\mathbf{x}\|=\left(\sum_{j=1}^{m} x_{j}^{2}\right)^{1 / 2}$. Let the sets $Z$ and $Z^{+}$denote the set of integers and the set of nonnegative integers, respectively. Let $A[n]$ be an $m \times m$ matrix whose entries $a_{i j}[n]$ are real functions defined in $Z_{n_{0}}^{+}=\left\{n_{0}, n_{0}+1, \ldots\right\}$ and $n_{0} \in Z^{+}$. For any $n \in Z_{n_{0}}^{+}$, the norm of matrix $A[n]$ is defined by

$$
\|A[n]\|=\max _{|x| \neq 0} \frac{\|A[n] x\|}{\|\mathbf{x}\|} .
$$

For convenience, the notation $|A|=\left(\left|a_{i j}\right|\right)_{m \times m}$ and $|B|=$ $\left(\left|b_{i j}\right|\right)_{m \times m}$.

For systems (15) and (16), the initial functions are given by

and

$$
\mathbf{x}[n]=\phi[n], \quad-\infty \leq n \leq n_{0}
$$

$$
\mathbf{x}[n]=\phi[n], \quad 0 \leq n \leq n_{0}
$$

respectively. The solution of system (15) [or system (16)] with initial values $\left(n_{0}, \phi\right)$ is denoted by $\mathbf{x}\left[n, n_{0}, \phi\right]$ and it satisfies (15) [or (16)] for $n>n_{0}$ and agrees with $\phi$ on the set $\left\{-\infty, \ldots, n_{0}\right\}$ (or $\left\{0,1, \ldots, n_{0}\right\}$ ).
Let $l^{\infty}\left(Z^{+}\right)$denote the set of all bounded sequences $\phi$ : $Z^{+} \rightarrow R^{m}$; given $\phi$ in $l^{\infty}\left(Z^{+}\right)$, define

$$
\|\phi\|_{n_{0}}=\max \left\{\|\phi[s]\|: s=0,1,2, \ldots, n_{0}\right\} .
$$

Let $l^{\infty}(Z)$ denote the set of all bounded sequences $\phi: Z \rightarrow$ $R^{m}$; given $\phi$ in $l^{\infty}(Z)$, define

$$
\|\phi\|_{n_{0}}=\sup \left\{\|\phi[s]\|:-\infty<s \leq n_{0}, s \in Z\right\} .
$$

Definition 2.1: Consider the system (16) with initial conditions $\left(n_{0}, \phi\right) \in Z^{+} \times l^{\infty}\left(Z^{+}\right)$; the trivial solution $x \equiv 0$ is called as follows.

1) Stable if given any $n_{0} \geq 0$ and $\varepsilon>0$; there exists a number $\delta>0\left(\delta=\delta\left(\varepsilon, n_{0}\right)\right)$ such that whenever $\|\phi\|_{n_{0}} \leq \delta$, the solution $\mathbf{x}\left[n, n_{0}, \phi\right]$ exists for $n \geq n_{0}$ and satisfies

$$
\left\|\mathbf{x}\left[n, n_{0}, \phi\right]\right\| \leq \varepsilon .
$$

Otherwise, the trivial solution $x \equiv 0$ is unstable.

2) Uniformly stable if it is stable and $\delta$ can be chosen independent of $n_{0}>0$.

3) Asymptotically stable if it is stable and if given any $\left(n_{0}, \phi\right)$ there exists $\eta\left(n_{0}\right)>0$, such that when $\|\phi\|_{n_{0}} \leq$ $\eta\left(n_{0}\right)$

$$
\mathbf{x}\left[n, n_{0}, \phi\right] \rightarrow 0 \quad \text { as } n \rightarrow \infty .
$$

4) Uniformly asymptotically stable if it is uniformly stable and if there exists $\eta>0$, such that given any $\varepsilon>0$ there exists $N(\varepsilon)>0$ such that

$$
\left\|\mathbf{x}\left[n+N(\varepsilon), n_{0}, \phi\right]\right\| \leq \varepsilon
$$

uniformly for all $n>n_{0}, n_{0} \geq 0$, and all $\phi \in l^{\infty}\left(Z^{+}\right)$, with $\|\phi\|_{n_{0}} \leq \eta$.

5) Exponentially stable if there exist two positive numbers $\alpha$ and $\lambda$ such that for sufficiently small $\|\phi\|_{n_{0}}$

$$
\|\mathbf{x}[n]\| \leq \alpha\|\phi\|_{n_{0}} e^{-\lambda\left(n-n_{0}\right)} \quad \forall \quad n \geq n_{0} .
$$

6) Globally asymptotically (uniformly asymptotically, exponentially) stable if 3) [4), 5) ] is satisfied for $\forall \phi \in$ $l^{\infty}\left(Z^{+}\right)$.

Let $\left(n_{0}, \phi\right)$ be a pair of initial data in $Z \times l^{\infty}(Z)$ for system (15), i.e., $\mathbf{x}[n]=\phi[n]$ on $n \in\left\{-\infty, \ldots,-1,0,1, \ldots, n_{0}\right\}$, $n \geq n_{0}$. The various stability properties for the trivial solution of system (15) can be defined in the same way as in Definition 2.1. Note that if $\mathbf{x}[n]$ is a solution of system (15), then

$$
\begin{aligned}
\mathbf{x}[n+N+1] & =A \mathbf{x}[n+N]+B \sum_{\nu=-\infty}^{n+N} K[n+N-\nu] G(\mathbf{x}[\nu]) \\
& =A \mathbf{x}[n+N]+B \sum_{\nu=-\infty}^{n} K[n-\nu] G(\mathbf{x}[\nu+N])
\end{aligned}
$$

for $N \in Z^{+}$. Thus, $\mathbf{x}[n+N]$ is also a solution of system (15). 
For any initial pair $\left(n_{0}, \phi\right)$, let $\phi_{n_{0}}[n]=\phi\left[n+n_{0}\right]$; since $\mathbf{x}\left[n, n_{0}, \phi\right]$ and $\mathbf{x}\left[n-n_{0}, 0, \phi_{n_{0}}\right]$ satisfy the same initial condition, it follows:

$$
\mathbf{x}\left[n, n_{0}, \phi\right]=\mathbf{x}\left[n-n_{0}, 0, \phi_{n_{0}}\right] .
$$

In this sense, we can say that system (15) is "autonomous." In particular, it follows that one needs only to consider system (15) with initial time $n_{0}=0$. Moreover, stability and uniform stability are equivalent in such cases.

\section{LINEAR APPROXIMATION AND LOCAL STABILITY}

It is a formalization of the intuition that a nonlinear system should behave similarly to its linearized approximation for small range motions. In this section, we will consider the properties of linear approximations of systems (15) and (16) with the assumption of $g_{i}(\cdot) \in \mathcal{S}$. Rewrite systems (15) and (16) as

$$
\mathbf{x}[n+1]=A \mathbf{x}[n]+B \sum_{\nu=-\infty}^{n} K[n-\nu](\ell \mathbf{x}[\nu]+h(\mathbf{x}[\nu]))
$$

and

$$
\mathbf{x}[n+1]=A \mathbf{x}[n]+B \sum_{\nu=0}^{n} K[n-\nu](\ell \mathbf{x}[\nu]+h(\mathbf{x}[\nu]))
$$

respectively, where

$$
\ell=\operatorname{diag}\left(\ell_{1}, \ell_{2}, \ldots, \ell_{m}\right)=\frac{\partial G}{\partial x}(0)
$$

and $h(x)$ stands for higher order terms in $x$, i.e.,

$$
h(x)=\left[\frac{\partial G}{\partial x}(\xi)-\frac{\partial G}{\partial x}(0)\right] x
$$

$\xi$ is a point on the linear segment connecting $x$ to the origin.

If we ignore the higher order terms in systems (18) and (19), then systems

$$
\mathbf{x}[n+1]=A \mathbf{x}[n]+\ell B \sum_{\nu=-\infty}^{n} K[n-\nu] \mathbf{x}[\nu]
$$

and

$$
\mathbf{x}[n+1]=A \mathbf{x}[n]+\ell B \sum_{\nu=0}^{n} K[n-\nu] \mathbf{x}[\nu]
$$

are the linearizations (or linear approximations) of systems (15) and (16) at the equilibrium point $x=0$, respectively. As the linear system (21) is autonomous, its stability and uniform stability are equivalent, which is closely related to uniform stability of (22). The following has been proved [17].

Theorem 3.1: Let $K[n] \in l^{1}\left(Z^{+}\right)$. Then, the following statements are equivalent:

1) the trivial solution of system (22) is uniformly stable;

2) the trivial solution of system (21) is (uniformly) stable.

Theorem 3.2: Let $K[n] \in l^{1}\left(Z^{+}\right)$and suppose that system (22) is uniformly stable. Then, the following statements are equivalent:
1) the trivial solution of system (22) is uniformly asymptotically stable;

2) the trivial solution of system (21) is (uniformly) asymptotically stable.

Based on Theorems 3.1 and 3.2, one only needs to consider system (22) since the results are also true for system (21) and vice versa.

We now state and establish the following result about local stability of the trivial solution for the nonlinear system (19) by investigating its stability of trivial solution for the linear approximation system (22).

Proposition 3.3: If $K[n]$ is in $l^{1}\left(Z^{+}\right)$and suppose that the trivial solution of system (22) is uniformly asymptotically stable, then the nonlinear system (19) is locally uniformly asymptotically stable.

To prove Proposition 3.3, we introduce here the so-called resolvent matrix of linear Volterra difference system (22), which is far more theoretical than practical and enables us to overcome some difficulties of dealing with a variety of initial conditions.

The resolvent matrix $R[n]$ associated with the linear system (22) satisfies the resolvent matrix equation

$$
R[n+1]=A R[n]+\ell B \sum_{\nu=0}^{n} R[\nu] K[n-\nu], \quad R(0)=I
$$

or equivalently

$$
R[n+1]=A R[n]+\ell B \sum_{\nu=0}^{n} R[n-\nu] K[\nu], \quad R(0)=I .
$$

For any initial value $\left(n_{0}, \phi\right)$, system (22) can be written as

$$
\begin{array}{r}
\mathbf{x}[n+1]=A \mathbf{x}[n]+\ell B \sum_{\nu=n_{0}}^{n} K[n-\nu] \mathbf{x}[\nu]+r[n], \\
n \geq n_{0} \geq 0
\end{array}
$$

where $r[n]=\ell B \sum_{\nu=0}^{n_{0}-1} K[n-\nu] \phi[\nu]$ if $n_{0} \geq 1$ and $r$ is related to the initial function $\phi$ and $r[n] \equiv 0$ when $n_{0}=0$.

Given any initial value $\left(n_{0}, \phi\right)$, by the variation of constant formula [17], the corresponding solution $\mathbf{x}\left[n, n_{0}, \phi\right]$ of system (22) can be written in the form of

$$
\begin{aligned}
\mathbf{x}\left[n, n_{0}, \phi\right]=R[n & \left.-n_{0}\right] \phi\left[n_{0}\right] \\
& +\ell B \sum_{\nu=n_{0}}^{n-1} R[n-\nu-1] \sum_{\tau=0}^{n_{0}-1} K[\nu-\tau] \phi[\tau] .
\end{aligned}
$$

Replacing $n$ in the previous formula with $n+n_{0}$; then, we have

$$
\begin{aligned}
\mathbf{x}\left[n+n_{0}, n_{0}, \phi\right]= & \left.R[n] \phi\left[n_{0}\right]+\ell B \sum_{\nu=n_{0}}^{n+n_{0}-1} R\left[n+n_{0}-\nu-1\right)\right] \\
& \cdot \sum_{\tau=0}^{n_{0}-1} K[\nu-\tau] \phi[\tau] \\
= & R[n] \phi\left[n_{0}\right]+\ell B \sum_{\nu=0}^{n-1} R([n-\nu-1] \\
& \cdot \sum_{\tau=0}^{n_{0}-1} K\left[\nu+n_{0}-\tau\right] \phi[\tau] .
\end{aligned}
$$


Lemma 3.4: Suppose that $B[n] \in l^{1}\left(Z^{+}\right)$and $R[n]$ is the resolvent of system (22). Then, the following statements hold true.

1) The trivial solution $\mathbf{x}[n] \equiv 0$ of system (22) is uniformly stable if and only if $\mathbf{y}[n]$ defined by

$$
\mathbf{y}[n]=\sum_{\tau=1}^{\infty} \sum_{\nu=0}^{n-1} R[n-\nu-1] K[\nu+\tau]
$$

is finite for all $n \in Z^{+}$and both $R[n]$ and $\mathbf{y}[n]$ are uniformly bounded on $Z^{+}$.

2) The trivial solution $\mathbf{x}[n] \equiv 0$ of system (22) is uniformly asymptotically stable if and only if it is uniformly stable and both $\|R[n]\|$ and $\|\mathbf{y}[n]\|$ tend to zero as $n \rightarrow \infty$.

Proof: Suppose that $\mathbf{x}[n] \equiv 0$ is uniformly stable. Then, there exists a constant $M$ such that for any $\left(n_{0}, \phi\right)$ with $n_{0} \geq 0$ and $\|\phi\|_{n_{0}} \leq 1$, one obtains

$$
\left\|\mathbf{x}\left[n+n_{0}, n_{0}, \phi\right]\right\| \leq M, \quad n \geq 0 .
$$

If $n_{0}=0$, then $\left\|\mathbf{x}\left[n+n_{0}, n_{0}, \phi\right]\right\|=\|\mathbf{x}[n, 0, \phi[0]]\|=$ $\|R[n] \phi[0]\| \leq M$ for all $n \geq 0$ and $\|\phi[0]\| \leq 1$. In particular, choosing $\phi[0]$ such that $\|R[n] \phi[0]\|=\|R[n]\|$, we have $\|R[n]\| \leq M$ for all $n \geq 0$. By the variation of constant formula (26)

$$
\begin{aligned}
\mathbf{x} & {\left[n+n_{0}, n_{0}, \phi\right]-R[n] \phi\left[n_{0}\right] } \\
& =\ell B \sum_{\nu=0}^{n-1} R[n-\nu-1]\left\{\sum_{\tau=0}^{n_{0}-1} K\left[\nu+n_{0}-\tau\right] \phi[\tau]\right\} \\
& =\ell B \sum_{\nu=0}^{n-1} R[n-\nu-1]\left\{\sum_{\tau=1}^{n_{0}} K[\nu+\tau] \phi\left[n_{0}-\tau\right]\right\} \\
& =\ell B \sum_{\tau=1}^{n_{0}}\left\{\sum_{\nu=0}^{n-1} R[n-\nu-1] K[\nu+\tau]\right\} \phi\left[n_{0}-\tau\right] .
\end{aligned}
$$

Applying property of triangle inequality to the previous equation, for all $n, n_{0} \geq 0$

$$
\begin{aligned}
\|\ell B\|\left\|\sum_{\tau=1}^{n_{0}} \sum_{\nu=0}^{n-1} R[n-\nu-1] K[\nu+\tau]\right\| \\
\leq\left\|\mathbf{x}\left[n+n_{0}, n_{0}, \phi\right]\right\|+\left\|R[n] \phi\left[n_{0}\right]\right\| \leq 2 M .
\end{aligned}
$$

Let $n_{0}$ tend to infinity, then $\|\mathbf{y}[n]\| \leq 2 M$ for all $n \geq 0$.

Conversely, if $\|R[n]\| \leq \eta$ and $\|\mathbf{y}[n]\|<\eta$ for some constant $\eta$, then by (26) one has

$$
\begin{aligned}
& \left\|\mathbf{x}\left[n+n_{0}, n_{0}, \phi\right]\right\| \\
& \leq\left\|R[n] \phi\left[n_{0}\right]\right\| \\
& \left.\quad+\|\ell B\| \| \sum_{\tau=1}^{n_{0}}\left\{\sum_{\nu=0}^{n-1} R[n-\nu-1] K[\nu+\tau)\right]\right\} \phi\left(\left[n_{0}-\tau\right] \|\right. \\
& \leq \eta\left\|\phi\left[n_{0}\right]\right\|+\|\ell B\| \eta\|\phi\|_{n_{0}} \leq \eta(1+\|\ell B\|)\|\phi\|_{n_{0}} .
\end{aligned}
$$

Thus, $x \equiv 0$ is uniformly stable. This proves condition 1 ). Condition 2) follows in a similar manner.
Lemma 3.5: [17] Suppose that $K[n] \in l^{1}\left(Z^{+}\right)$. The trivial solution of system (22) is uniformly asymptotically stable if and only if either one of the following conditions are satisfied:

1) $\operatorname{det}(z I-A-\ell B \tilde{K}(z)) \neq 0$ for $|z| \geq 1$;

2) $R[n] \in l^{1}\left(Z^{+}\right)$

where $R[n]$ is the resolvent of system (22) and $\tilde{K}$ is the $Z$-transform of $K[n]$.

We are now in a position to prove Proposition 3.3 as follows.

Proof: Suppose that linear system (22) is uniformly stable. Then, by Lemmas 3.4 and 3.5, $R[n] \in l^{1}\left(Z^{+}\right)$and $\mathbf{x}[n+$ $\left.n_{0}, n_{0}, \phi\right] \in l^{1}\left(Z^{+}\right)$, where $x\left(n, n_{0}, \phi\right)$ is the solution of system (22).

System (19) can be rearranged as

$$
\begin{aligned}
\mathbf{x}[n+1]= & A \mathbf{x}[n]+B \ell \sum_{\nu=0}^{n} K[n-\nu] \mathbf{x}[\nu] \\
& +B \sum_{\nu=0}^{n_{0}-1} K[n-\nu] h(\phi[\nu]) \\
& +B \sum_{\nu=n_{0}}^{n} K[n-\nu] h(\mathbf{x}[\nu]) .
\end{aligned}
$$

Suppose that now $\mathbf{z}\left[n, n_{0}, \phi\right]$ is the solution of system (19) with the same initial value $\left(n_{0}, \phi\right)$ of the solution $x\left[n, n_{0}, \phi\right]$ of system (22). Employing the formula of variation of constants again, $\left.\mathbf{z}\left[n, n_{0}, \phi\right)\right]$ can be expressed as

$$
\begin{aligned}
\mathbf{z}\left[n, n_{0}, \phi\right]= & \mathbf{x}\left[n, n_{0}, \phi\right] \\
& +B \sum_{\nu=n_{0}}^{n-1} R[n-\nu-1] \sum_{\tau=0}^{n_{0}-1} K[\nu-\tau] h(\phi[\tau]) \\
& +B \sum_{\nu=n_{0}}^{n-1} R[n-\nu-1] \sum_{\tau=n_{0}}^{\nu} K[\nu-\tau] h(z[\tau]) .
\end{aligned}
$$

Replacing $n$ by $n+n_{0}$, we have

$$
\begin{aligned}
\mathbf{z}[n & \left.+n_{0}, n_{0}, \phi\right] \\
= & \mathbf{x}\left[n+n_{0}, n_{0}, \phi\right] \\
& +B \sum_{\nu=0}^{n-1} R[n-\nu-1]\left\{\sum_{\tau=1}^{n_{0}} K[\nu+\tau] h\left(\phi\left(\left[n_{0}-\tau\right]\right)\right\}\right. \\
& \left.+B \sum_{\nu=0}^{n-1} R[n-\nu-1]\left\{\sum_{\tau=0}^{\nu} K[\nu-\tau] h\left(\mathbf{z}\left[n_{0}+\tau\right)\right]\right)\right\} \\
= & \mathbf{x}\left[n+n_{0}, n_{0}, \phi\right] \\
& +B \sum_{\tau=1}^{n_{0}}\left\{\sum _ { \nu = 0 } ^ { n - 1 } R [ n - \nu - 1 ] K ( [ \nu + \tau ] \} h \left(\phi\left(\left[n_{0}-\tau\right]\right)\right.\right. \\
& +B \sum_{\tau=0}^{n-1}\left\{\sum_{\nu=\tau}^{n-1} R[n-\nu-1] K[\nu-\tau]\right\} \times h\left(\mathbf{z}\left[n_{0}+\tau\right]\right) .(28)
\end{aligned}
$$

Let $y[n]$ be defined as in Lemma 3.4. Then, from the proof of Lemma 3.4, $\mathbf{y}[n] \in l^{1}\left(Z^{+}\right)$, as $R[n]$ and $x\left(\left[n+n_{0}, n_{0}, \phi\right]\right.$ are both in $l^{1}\left(Z^{+}\right)$.

From

$$
\frac{\|h(\mathbf{x})\|}{\|\mathbf{x}\|} \rightarrow 0 \quad \text { as } \quad\|\mathbf{x}\| \rightarrow 0
$$


then, for any

$$
0<\gamma<\frac{1}{\|B\| \sum_{n=0}^{\infty} \mathbf{y}[n]}
$$

there exists $r>0$ such that

$$
\|h(\mathbf{x})\|<\gamma\|\mathbf{x}\| \quad \forall\|\mathbf{x}\|<r .
$$

Summing up (28) over $n$ from $n=0$ to $n=\infty$ and introducing $\alpha[n, \nu, \tau]=R[n-\nu-1] K[\nu+\tau]$, we have

$$
\begin{aligned}
& \left.\sum_{n=0}^{\infty} \| \mathbf{z}\left[n+n_{0}\right)\right] \| \\
& \leq \sum_{n=0}^{\infty}\left\|\mathbf{x}\left[n+n_{0}\right]\right\|+\gamma\|B\| \sum_{n=0}^{\infty}\left\|\sum_{\tau=1}^{n_{0}}\left\{\sum_{\nu=0}^{n-1} \alpha[n, \nu, \tau]\right\}\right\| \phi \|_{n_{0}} \\
& \quad+\gamma\|B\| \sum_{n=0}^{\infty}\left\|\sum_{\tau=1}^{n_{0}}\left\{\sum_{\nu=0}^{n-1} \alpha[n, \nu, \tau]\right\}\right\| z\left(n+n_{0}\right) \| \\
& \leq \sum_{n=0}^{\infty}\|R[n]\|\left\|\phi\left[n_{0}\right]\right\|+(\|\ell\|+\gamma)\|B\|\left(\sum_{n=0}^{\infty}\|\mathbf{y}[n]\|\right)\|\phi\|_{n_{0}} \\
& \quad+\gamma\|B\|\left(\sum_{n=0}^{\infty}\|\mathbf{y}[n]\|\right) \sum_{n=0}^{\infty}\left\|\mathbf{z}\left[n+n_{0}\right]\right\| .
\end{aligned}
$$

Isolating $\sum_{n=0}^{\infty}\left\|\mathbf{z}\left[n+n_{0}\right]\right\|$ from (29) yields

$$
\sum_{n=0}^{\infty}\left\|\mathbf{z}\left[n+n_{0}\right]\right\| \leq D\|\phi\|_{n_{0}}
$$

where $z\left(n+n_{0}\right)=z\left(n+n_{0}, n_{0}, \phi\right)$ and

$$
D=\frac{\left\{\sum_{n=0}^{\infty}\|R[n]\|+(\|\ell\|+\gamma)\|B\|\left(\sum_{n=0}^{\infty}\|\mathbf{y}[n]\|\right)\right\}}{1-\gamma\|B\|\left(\sum_{n=0}^{\infty}\|\mathbf{y}[n]\|\right)} .
$$

By Lemmas 3.4 and 3.5, $R[n], \mathbf{y}[n] \in l^{1}\left(Z^{+}\right)$. It follows that $\mathbf{z}\left[n+n_{0}\right] \in l^{1}\left(Z^{+}\right)$. Thus, for any $\varepsilon>0$, there exists a constant $T>0$ such that

$$
\left\|\mathbf{z}\left[n+n_{0}, n_{0}, \phi\right]\right\|<\varepsilon
$$

for all $n \geq T+n_{0}$, which implies that the trivial solution of the nonlinear system (19) is locally uniformly asymptotically stable.

\section{Global Stability}

In this section, assuming that $g_{i}(\cdot) \in \mathcal{G} \mathcal{L}$, we give several sufficient conditions on global asymptotic stability of the trivial solution for the neural network model (12).

Proposition 4.1: Suppose that there exist positive constants $r_{1}, r_{2}, \ldots, r_{m}$ such that

$$
\left|a_{j}\right|+\bar{\ell}_{j} \sum_{i=1}^{m} \frac{r_{i}}{r_{j}}\left|b_{i j}\right|\left(\sum_{\nu=0}^{\infty}\left|k_{i j}[\nu]\right|\right)<1
$$

for all $j=1,2, \ldots, m$. Then, the trivial solution of system (12) is globally asymptotically stable.
Proof: From (12), exchanging with $n-\nu$ and $\nu$, we have

$$
\begin{array}{r}
x_{i}[n+1]=a_{i} x_{i}[n]+\sum_{j=1}^{m} b_{i j} \sum_{\nu=0}^{\infty} k_{i j}[\nu] g_{j}\left(x_{j}[n-\nu]\right), \\
i=1,2, \ldots, m
\end{array}
$$

thus

$$
\begin{array}{r}
\left|x_{i}[n+1]\right| \leq\left|a_{i}\right|\left|x_{i}[n]\right|+\sum_{j=1}^{m}\left|b_{i j}\right| \sum_{\nu=0}^{\infty}\left|k_{i j}[\nu]\right| \bar{\ell}_{j}\left|x_{j}[n-\nu]\right| \\
i=1,2, \ldots, m .
\end{array}
$$

Let $V_{i}^{(1)}(\mathbf{x})[n]=\left|x_{i}[n]\right|$, then

$$
\begin{aligned}
& \triangle V_{i}^{(1)}(\mathbf{x})[n] \\
& =\left|x_{i}[n+1]\right|-\left|x_{i}[n]\right| \\
& \leq\left(\left|a_{i}\right|-1\right)\left|x_{i}[n]\right|+\sum_{j=1}^{m}\left|b_{i j}\right| \bar{\ell}_{j} \sum_{\nu=0}^{\infty}\left|k_{i j}[\nu]\right|\left|x_{j}[n-\nu]\right| .
\end{aligned}
$$

Let

$$
V_{i}^{(2)}(\mathbf{x})[n]=\sum_{j=1}^{m}\left|b_{i j}\right| \bar{\ell}_{j}\left[\sum_{\nu=0}^{\infty}\left|k_{i j}[\nu]\right|\left(\sum_{p=n-\nu}^{n-1}\left|x_{j}[p]\right|\right)\right]
$$

then

$$
\begin{aligned}
& \triangle V_{i}^{(2)}(\mathbf{x})[n] \\
& =\sum_{j=1}^{m}\left|b_{i j}\right| \bar{\ell}_{j}\left(\sum_{\nu=0}^{\infty}\left|k_{i j}[\nu]\right|\right)\left(\left|x_{j}[n]\right|-\left|x_{j}[n-\nu]\right|\right) .
\end{aligned}
$$

Define a Liapunov functional $V(\mathbf{x})[n]$ as

$$
V[n]=\sum_{i=1}^{m} r_{i}\left(V_{i}^{(1)}(\mathbf{x})[n]+V_{i}^{(2)}(\mathbf{x})[n]\right) .
$$

Using (34) and (35) and calculating $\triangle V[n]=V[n+1]-V[n]$ along the solution of system (12), one obtains

$$
\begin{aligned}
\triangle V[n] & \sum_{i=1}^{m} r_{i}\left\{\left(\left|a_{i}\right|-1\right)\left|x_{i}[n]\right|+\sum_{j=1}^{m}\left|b_{i j}\right| \bar{\ell}_{j}\left(\sum_{\nu=0}^{\infty}\left|k_{i j}[\nu]\right|\right)\left|x_{j}[n]\right|\right\} \\
= & \sum_{i=1}^{m} r_{i}\left(\left|a_{i}\right|-1\right)\left|x_{i}[n]\right| \\
& +\sum_{i=1}^{m} r_{i} \sum_{j=1}^{m}\left|b_{i j}\right| \bar{\ell}_{j}\left(\sum_{\nu=0}^{\infty}\left|k_{i j}[\nu]\right|\right)\left|x_{j}[n]\right| \\
= & \sum_{j=1}^{m} r_{j}\left(\left|a_{j}\right|-1\right)\left|x_{j}[n]\right| \\
& +\sum_{j=1}^{m} \sum_{i=1}^{m} r_{i}\left|b_{i j}\right| \bar{\ell}_{j}\left(\sum_{\nu=0}^{\infty}\left|k_{i j}[\nu]\right|\right)\left|x_{j}[n]\right| \\
= & -\sum_{j=1}^{m}\left\{r_{j}\left(1-\left|a_{i}\right|\right)-\sum_{i=1}^{m} r_{i}\left|b_{i j}\right| \bar{\ell}_{j} \sum_{\nu=0}^{\infty}\left|k_{i j}[\nu]\right|\right\}\left|x_{j}[n]\right| \\
\leq & -\sum_{j=1}^{m} e_{j}\left|x_{j}[n]\right|
\end{aligned}
$$

where $e_{j}=r_{j}\left(1-\left|a_{i}\right|\right)-\sum_{i=1}^{m} r_{i}\left|b_{i j}\right| \bar{\ell}_{j} \sum_{\nu=0}^{\infty}\left|k_{i j}[\nu]\right|>0$ due to condition (31). 
The inequalities in (37) lead to

$$
\left\{\begin{array}{l}
V(\mathbf{x})[n+1]-V(\mathbf{x})[n] \leq-\sum_{j=1}^{m} e_{j}\left|x_{j}[n]\right| \\
V(\mathbf{x})[n]-V(\mathbf{x})[n-1] \leq-\sum_{j=1}^{m} e_{j}\left|x_{j}[n-1]\right| \\
\ldots \ldots \ldots \ldots \ldots \ldots \ldots \ldots \ldots \ldots \ldots \ldots \ldots \ldots \ldots \ldots \ldots \ldots \ldots \ldots \\
V(\mathbf{x})[1]-V(\mathbf{x})[0] \leq-\sum_{j=1}^{m} e_{j}\left|x_{j}[0]\right| .
\end{array}\right.
$$

Adding the inequalities in (38) vertically, we have

$$
V(\mathbf{x})[n+1]+\sum_{j=1}^{m} e_{j} \sum_{\nu=0}^{n}\left|x_{j}[\nu]\right| \leq V(\mathbf{x})[0]
$$

It follows from (36) and (39) that

$$
\sum_{\nu=0}^{n}\left|x_{j}[\nu]\right|
$$

uniformly converges as $n \rightarrow \infty$. Consequently, we can conclude that

$$
|\mathbf{x}[n]| \rightarrow 0
$$

uniformly as $n \rightarrow \infty$. Thus, the globally asymptotic stability of the trivial solution of system (12) follows.

Proposition 4.2: Suppose that there exist positive constants $r_{1}, r_{2}, \ldots, r_{m}$ such that

$$
\left|a_{i}\right|+\sum_{j=1}^{m} \frac{r_{j}}{r_{i}} \bar{\ell}_{j}\left|b_{i j}\right|\left(\sum_{n=0}^{\infty}\left|k_{i j}[n]\right|\right)<1
$$

for all $i=1,2, \ldots, m$. Then, the trivial solution of system (12) is globally asymptotically stable.

Proof: Let $P=\operatorname{diag}\left(r_{1}, r_{2}, \ldots, r_{m}\right)$ and

$$
C=I-\left(|A|+|B| \bar{L} \odot \sum_{\nu=0}^{\infty}|K[\nu]|\right)
$$

where $\bar{L}=\operatorname{diag}\left(\bar{\ell}_{1}, \bar{\ell}_{2}, \ldots, \bar{\ell}_{m}\right)$ and " $\odot "$ represents the element product of two matrices. From the condition (40), we have

$$
r_{i}\left|a_{i}\right|+\sum_{j=1}^{m} r_{j} \bar{\ell}_{j}\left|b_{i j}\right|\left(\sum_{\nu=0}^{\infty}\left|k_{i j}[\nu]\right|\right)<r_{i}
$$

for some constants $r_{i}$ and for all $i=1,2, \ldots, m$. It indicates that the matrix $P C$ is strictly diagonal row dominant. Notice also that matrix $C$ has nonpositive off-diagonal elements. From $M$-matrix theory [2], there exists a diagonal matrix $P$ (for convenience and no ambiguity, we still use $P$ ) such that $C P$ is strictly diagonal column dominant and then the condition (31) of Proposition 4.1 is satisfied. This completes the proof.

As the nondelay items $b_{i i} k_{i i}[0] g_{j}\left(x_{j}[n]\right)$ in the convolution sum of system (12) can contribute to the diagonal domination, through a simple modification to the proofs of the previous propositions, we have Proposition 4.3.

Proposition 4.3: Suppose that for all $j=1,2, \ldots, m$ there exist $r_{1}, r_{2}, \ldots, r_{m}$, such that (42), shown at the bottom of the page, holds, or for all $i=1,2, \ldots, m$, there exist $r_{1}, r_{2}, \ldots, r_{m}$, such that (43), shown at the bottom of the page, holds. Then, the trivial solution of system (12) is globally asymptotically stable.

For linear systems, one can verify that the stronger the delay kernel of $K[s]$ is, the stronger the stability conclusion becomes [17]. For the nonlinear neural network system (12), we have the following global exponential stability result.

Proposition 4.4: Suppose that there exits a $\lambda>1$, such that $\left\{\lambda^{n} K[n]\right\}$ is in $l^{1}\left(Z^{+}\right)$and any of the conditions of Propositions $4.1-4.3$ holds. Then, the trivial solution of system (12) is globally exponentially stable.

Proof: We only prove here for the condition (31) of the Proposition 4.1 and the proofs for other conditions are similar.

Since $\lambda^{n} K[n] \in l^{1}\left(Z^{+}\right)$, we have

$$
\sum_{\nu=0}^{\infty} \lambda^{\nu}\left|k_{i j}[\nu]\right|<\infty
$$

Let

$$
h(\lambda)=r_{j}\left(\frac{1}{\lambda}-\left|a_{j}\right|\right)-\bar{\ell}_{j} \sum_{i=1}^{m} r_{i}\left|b_{i j}\right| \sum_{\nu=0}^{\infty} \lambda^{\nu}\left|k_{i j}[\nu]\right| .
$$

$$
\max _{\underline{\ell}_{j} \leq \ell_{j} \leq \bar{\ell}_{j}}\left|a_{j}+\ell_{j} b_{j j} k_{j j}[0]\right|+\bar{\ell}_{j}\left|b_{j j}\right|\left(\sum_{\nu=1}^{\infty}\left|k_{j j}[\nu]\right|\right)+\bar{\ell}_{j} \sum_{i \neq j} \frac{r_{i}}{r_{j}}\left|b_{i j}\right|\left(\sum_{\nu=0}^{\infty}\left|k_{i j}[\nu]\right|\right)<1
$$

$$
\max _{\underline{\ell}_{i} \leq \ell_{i} \leq \bar{\ell}_{i}}\left|a_{i}+\ell_{i} b_{i i} k_{i i}[0]\right|+\bar{\ell}_{i}\left|b_{i i}\right|\left(\sum_{\nu=1}^{\infty}\left|k_{i i}[\nu]\right|\right)+\sum_{j \neq i} \frac{r_{j}}{r_{i}} \bar{\ell}_{j}\left|b_{i j}\right|\left(\sum_{\nu=0}^{\infty}\left|k_{i j}[\nu]\right|\right)<1
$$


Then, $h(\lambda)$ is well defined and

$$
h(1)=r_{j}\left(1-\left|a_{j}\right|\right)-\bar{\ell}_{j} \sum_{i=1}^{m} r_{i}\left|b_{i j}\right| \sum_{\nu=0}^{\infty} \lambda^{\nu}\left|k_{i j}[\nu]\right|=e_{j}>0
$$

from (31). By the continuity of $h(\lambda)$ for $\lambda \geq 1$, there exists $1<\eta<\lambda$, such that $h(\eta)>0$.

Choose a Liapunov function defined by (46), shown at the bottom of the page. Then, the equation shown at the bottom of the page, holds. Therefore

$$
V(\mathbf{x})[n] \leq V(\mathbf{x})[0] .
$$

However

$$
\begin{aligned}
V(\mathbf{x})[0] & \\
& =\sum_{i=0}^{m} r_{i}\left\{\left|x_{i}[0]\right|+\sum_{i=1}^{m} r_{i}\left|b_{i j}\right| \bar{\ell}_{j}\left(\sum_{\nu=0}^{\infty} \eta^{\nu}\left|k_{i j}[\nu]\right|\right) \sum_{p=-\nu}^{-1}\left|x_{j}[p]\right|\right\} \\
& \leq \sum_{i=0}^{m} r_{i}\left\{\left|\phi_{i}[0]\right|+\sum_{i=1}^{m} r_{i}\left|b_{i j}\right| \bar{\ell}_{j}\left(\sum_{\nu=0}^{\infty} \eta^{\nu}\left|k_{i j}[\nu]\right|\right)\left\|\phi_{i}\right\|_{0}\right\} \\
& \leq \sum_{j=0}^{m} r_{i}\left\{1+\sum_{i=1}^{m} r_{i}\left|b_{i j}\right| \bar{\ell}_{j}\left(\sum_{\nu=0}^{\infty} \eta^{\nu}\left|k_{i j}[\nu]\right|\right)\right\}\left\|\phi_{i}\right\|_{0} \\
& \leq M\|\phi\|_{0}
\end{aligned}
$$

where

$$
M=\sum_{j=0}^{m} r_{i}\left\{1+\sum_{i=1}^{m} r_{i}\left|b_{i j}\right| \bar{\ell}_{j}\left(\sum_{\nu=0}^{\infty} \eta^{\nu}\left|k_{i j}[\nu]\right|\right)\right\}
$$

are bounded.

From (46), we have

$$
\eta^{n} \sum_{i=1}^{m} r_{i}\left|x_{i}[n]\right| \leq M\|\phi\|_{0}
$$

i.e.,

$$
\sum_{i=1}^{m}\left|x_{i}[n]\right| \leq\left(\frac{1}{\eta}\right)^{n} \frac{M}{r}\|\phi\|_{0}
$$

where $r=\max _{1 \leq i \leq m}\left\{r_{i}\right\}$; so

$$
\left|x_{i}(\mathbf{x})\right| \rightarrow 0
$$

exponentially as $n \rightarrow \infty$, which implies that the trivial solution of system (12) is globally exponentially stable.

So far we have only focused on system (12) because of its "autonomous" property. The equivalence of the stability of systems (12) and (13) (see Theorems 3.1 and 3.2) indicates that the results are also true for system (13). For the special case of system (11), which is a first-order difference system, one can choose the delayed kernels as discrete $\delta$-function, as mentioned in Section II, and can easily verify that (31) of Proposition 4.1, (40) of Proposition 4.2, and (42)-(43) of Proposition 4.3 recover the following group of conditions that were given in [10] and [13] and each of them is sufficient for global exponential stability of system (11):

$$
\begin{array}{r}
\left|a_{j}\right|+\bar{\ell}_{j} \sum_{i=1}^{m}\left|b_{i j}\right|<1 \\
\left|a_{i}\right|+\sum_{j=1}^{m} \bar{\ell}_{i}\left|b_{i j}\right|<1 \\
\left|a_{j}\right|+\bar{\ell}_{j} \sum_{i=1}^{m} \frac{r_{i}}{r_{j}}\left|b_{i j}\right|<1 \\
\left|a_{i}\right|+\sum_{j=1}^{m} \frac{r_{j}}{r_{i}} \bar{\ell}_{i}\left|b_{i j}\right|<1 \\
\max _{\underline{\ell}_{j} \leq \ell_{j} \leq \bar{\ell}_{j}}\left|a_{j}+\ell_{j} b_{j j}\right|+\bar{\ell}_{j} \sum_{i \neq j} \frac{r_{i}}{r_{j}}\left|b_{i j}\right|<1 \\
\max _{\underline{\ell}_{i} \leq \ell_{i} \leq \bar{\ell}_{i}}\left|a_{i}+\ell_{i} b_{i i}\right|+\sum_{j \neq i} \frac{r_{j}}{r_{i}} \bar{\ell}_{j}\left|b_{i j}\right|<1 .
\end{array}
$$

Remark 4.5: The assumption of $g(\cdot) \in \mathcal{G} \mathcal{L}$ in this section is only for our convenience to state the propositions. In fact, the conclusions are also true for $g_{i}(\cdot) \in \mathcal{L}$ except for Proposition 4.3 , where we have to separate the self-regulation feedback from the convolution, and thus, monotonicity of the activity function is needed.

$$
V(\mathbf{x})[n]=\sum_{j=1}^{m} r_{i}\left\{\eta^{n}\left|x_{i}[n]\right|+\sum_{j=1}^{m}\left|b_{i j}\right| \bar{\ell}_{i}\left[\sum_{\nu=0}^{\infty} \eta^{\nu} \times\left|k_{i j}[\nu]\right|\left(\sum_{p=n-\nu}^{n-1} \eta^{p+1}\left|x_{j}[p]\right|\right)\right]\right\}
$$

$$
\begin{aligned}
\triangle V(\mathbf{x})[n] & \leq \sum_{i=1}^{m} r_{i}\left\{\eta^{n}\left(\eta\left|a_{i}\right|-1\right)\left|x_{i}[n]\right|+\eta^{n+1} \sum_{j=1}^{m}\left|b_{i j}\right| \bar{\ell}_{j}\left(\sum_{\nu=0}^{\infty} \eta^{\nu}\left|k_{i j}[\nu]\right|\right)\left|x_{j}[n]\right|\right\} \\
& =-\eta^{n+1} \sum_{j=1}^{m}\left\{r_{j}\left(\frac{1}{\eta}-\left|a_{i}\right|\right)-\sum_{i=1}^{m} r_{i}\left|b_{i j}\right| \bar{\ell}_{j}\left(\sum_{\nu=0}^{\infty} \eta^{\nu}\left|k_{i j}[\nu]\right|\right)\right\}\left|x_{j}[n]\right| \\
& =-\eta^{n+1} \sum_{j=1}^{m} h(\eta)\left|x_{j}[n]\right| \leq 0 .
\end{aligned}
$$




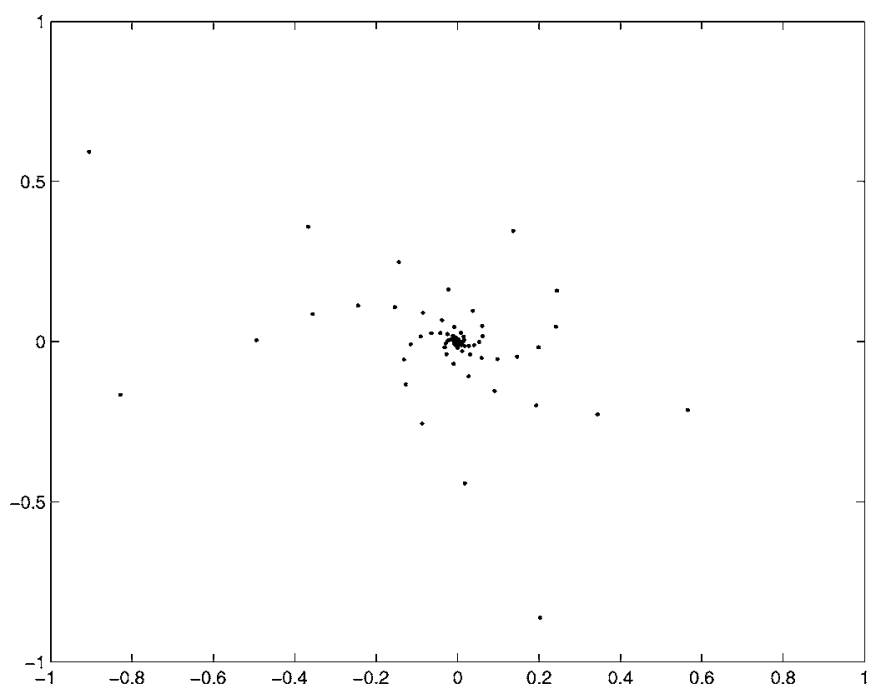

(a)

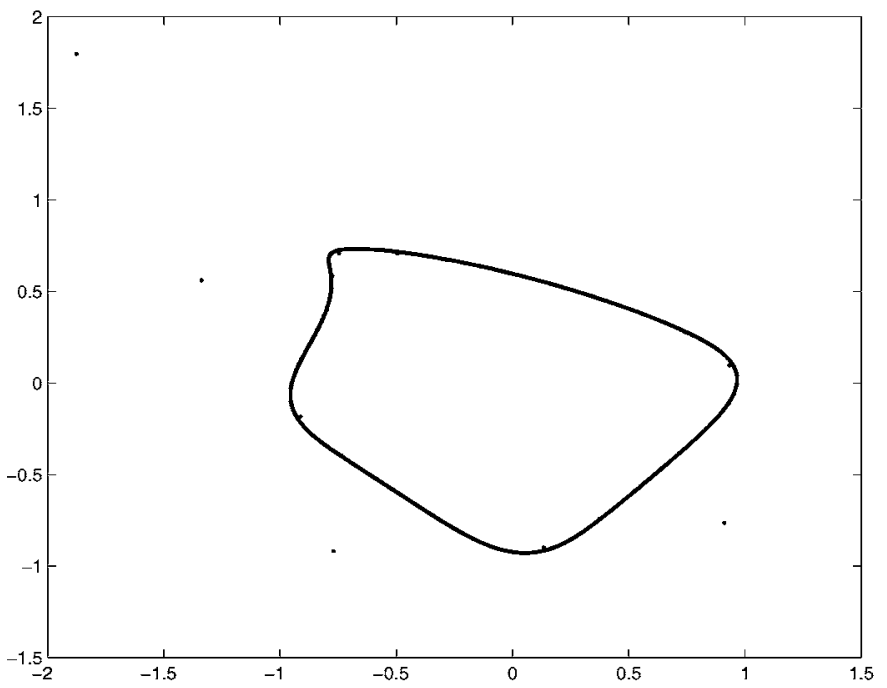

(c)

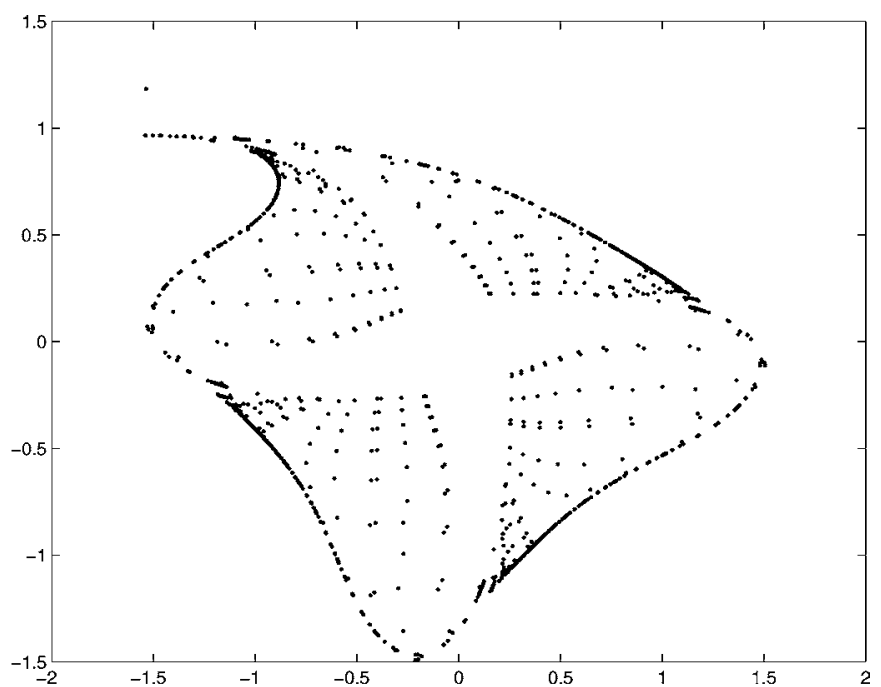

(b)

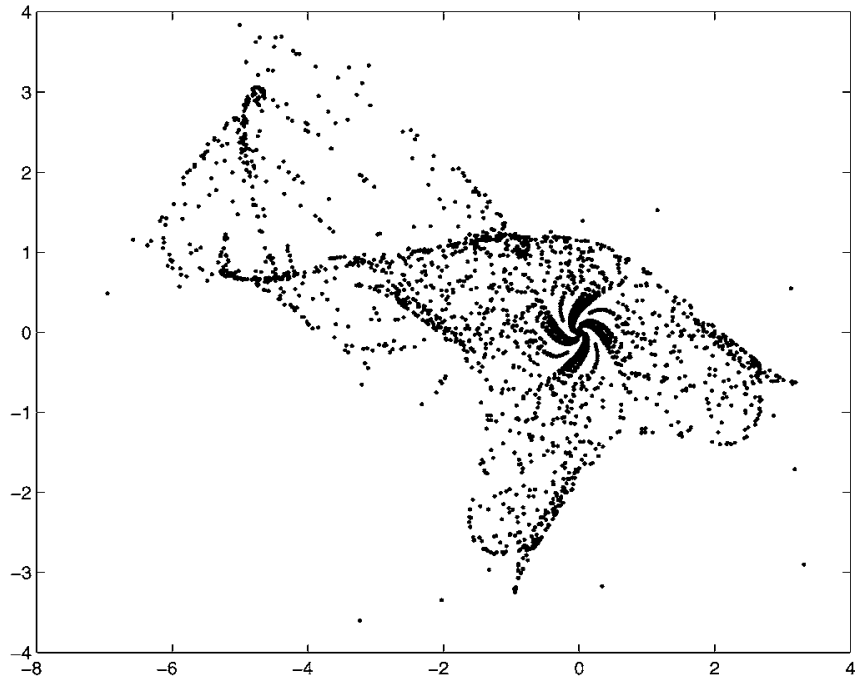

(d)

Fig. 1. Simulation of (53). (a) Asymptotically stable. (b) Starfish. (c) Periodic. (d) Chaotic.

\section{Discrete-Time Neural Networks Versus Their CONTINUOUS-TIME COUNTERPARTS}

The systems (12) and (13) that we have studied in previous sections can be considered as stand alone discrete-time neural networks or as discrete-time analogs of their continuous-time counterparts. In contradistinction to their continuous-time counterparts, even for simple discrete-time neural networks it is possible to observe some interesting behavior, including limit cycles and chaos. The following example is a discrete-time neural network, which we modified from [15] to the form of system (12) with delay kernel $k_{i j}[n]$ as discrete $\delta$-function $\delta[n]$.

1) Example 5.1: Consider the discrete-time neural network

$$
\left\{\begin{array}{l}
x_{1}[n+1]=a_{1} x_{1}[n]+b_{11} g_{1}\left(x_{1}[n]\right)+b_{12} g_{2}\left(x_{2}[n]\right) \\
x_{2}[n+1]=a_{2} x_{2}[n]+b_{21} g_{1}\left(x_{1}[n]\right)+b_{22} g_{2}\left(x_{2}[n]\right)
\end{array}\right.
$$

where

$$
\left\{\begin{array}{l}
g_{1}(\mathbf{x})=x-\left[\tanh \left(v_{1}(x+1.89)\right)-\tanh \left(1.89 v_{1}\right)\right] \\
g_{2}(\mathbf{x})=x+\left[\tanh \left(v_{2}(x-1.03)\right)-\tanh \left(1.03 v_{2}\right)\right]
\end{array}\right.
$$

and $\left[a_{1}, a_{2}\right]=[\pi-0.1,1.1]$ and $\left[\begin{array}{ll}b_{11} & b_{12} \\ b_{21} & b_{22}\end{array}\right]=\left[\begin{array}{rr}-\pi & 1 \\ -1 & -1\end{array}\right]$. The result of the simulation is plotted in the $x_{1}-x_{2}$ phase plane by choosing $\left[v_{1}, v_{2}\right]=[0.5,1.5],\left[v_{1}, v_{2}\right]=[1.23456,2.23456]$, $\left[v_{1}, v_{2}\right]=[1,2]$, and $\left[v_{1}, v_{2}\right]=[2,3]$, respectively. One can see the attractor changing from single point to a shape of starfish, to a stable limit cycle, and to chaos (Fig. 1).

One can either design a discrete-time neural network from scratch or discretize a given continuous-time system. The second approach has the advantage that one can keep track of the known properties of the continuous-time model. In practice, this type of approximation occurs when numerical integration algorithm is used for computer simulation, as in the Euler or Runge-Kutta methods. Of course, taking a differential model as the source of a difference model, one should check whether the discrete-time system still has the desired properties of its continuous-time counterpart. From the previous example, we can see that discrete-time version of a continuous-time system can posses complex dynamic behaviors.

Taking systems (12) and (13) as the discretization of systems (6) and (7) in the case of Euler's approximation with step-size 
$h$, one can easily check out that the conditions of global stability in Section IV are well coincident with the conditions assigned to systems (6) and (7) when $h$ is sufficiently small. For example, the condition (47) to system (11) is

$$
\left|1-\frac{h}{\tau_{j}}\right|+\bar{\ell}_{j} \sum_{i=1}^{m} \frac{h}{\tau_{i}}\left|w_{i j}\right|<1 .
$$

If $h<\tau_{i}$, then (54) becomes

$$
\frac{1}{\tau_{j}}>\bar{\ell}_{j} \sum_{i=1}^{m} \frac{1}{\tau_{i}}\left|w_{i j}\right|
$$

which is one of the simple sufficient conditions to guarantee global asymptotic stability of the trivial solution of system (11) [10], [19]. The remaining conditions of the group can be verified in the same way.

It is worth mentioning that if $w_{i j}=w_{j i}$ in system (4) is symmetric, the dynamic of system (4) is always convergent, namely, every initial state will approach a fixed point asymptotically. But, its discrete-time counterpart (11) is either convergent or approaches a periodic orbit of 2 (i.e., a two-cycle) [3], [7], [14]. To guarantee the convergent to fixed points (cycle-free), a sufficient condition can be obtained if the discretization step-size $h$ is small enough when the Euler's method is used.

When discretizing a continuous system, extra parameter (step-size, for example) is introduced into the discrete-time system. Therefore, it is no surprise that extra conditions are needed to keep the discrete-time system dynamically consistent with the continuous-time one.

In order to guarantee the stability of a discrete-time system under the same conditions with its continuous-time counterpart, we propose an unconventional finite difference method, which is specially useful for discrete-time neural network models and is stated as follows.

For systems (6) and (7), it is reasonable from sampling to replace the convolution integral in the right of the equation by a discrete convolution sum, i.e.,

$$
\int_{s} k_{i j}(t-s) g_{j}\left(x_{j}(s)\right) d s \Rightarrow \sum_{v} k_{i j}(t-v h) g_{i}\left(x_{j}[v h]\right)
$$

where $h$ is the sampling period or discrete step.

Recall the domination of the linear part in the stability of a continuous-time system; we consider the linear differential equation

$$
\frac{d x(t)}{d t}=-a x(t), a>0
$$

which is exactly solvable. For any step $h>0$, integration of (57) on $[t, t+h]$ leads to

$$
x(t+h)=e^{-a h} x(t) .
$$

Rearrange (58) to

$$
\frac{x(t+h)-x(t)}{\left(\frac{1-e^{-a h}}{a}\right)}=-a x(t) .
$$

Drawing inspiration from (57) and (59), we propose the following replacement:

$$
\frac{d x(t)}{d t} \Rightarrow \frac{x(t+h)-x(t)}{\Phi(h)}
$$

which may be used to eliminate the elementary numerical instability in the discrete-time modeling of a continuous-time differential equation. For many situations, $\Phi(h)$ also satisfies the condition

$$
\Phi(h)=h+O(h) .
$$

Let $t \triangleq n h$ and $x[n] \triangleq x(n h)$. Then, the discrete-time analog of system (6) or (7) can be written in the following form:

$$
\begin{aligned}
x_{i}[n+1]=(1 & \left.-\frac{\Phi(h)}{\tau_{i}}\right) x_{i}[n] \\
& +\frac{\Phi(h)}{\tau_{i}} \sum_{i=1}^{m} w_{i j} \sum_{v} k_{i j}[n-v] g_{j}\left(x_{j}[n]\right)
\end{aligned}
$$

where

$$
\Phi(h)=\tau_{i}\left(1-e^{-h / \tau_{i}}\right)
$$

or

$$
\Phi(h)=\frac{\tau_{i} h}{\tau_{i}+h}
$$

are the two common choices of $\Phi(h)$. Notice that the method of differential equations with piecewise constant arguments has been used to discretize continuous-time population models [18] and neural network models [22]. The method provided in this paper is based on sampling and instantaneous domination of the systems.

It is easy to verify that the stability conditions in Section IV to system (62) coincide well with the stability conditions to continuous-time systems (6) and (7) [10], [19], regardless of the size of $h$. In the following, we use computer simulation for an example to compare the Euler method and the unconventional finite difference method.

2) Example 5.2: Consider the neural network (6) with $m=$ $2, \tau_{1}=1 / 7, \tau_{2}=1 / 11$, and $\left[w_{i j}\right]=\left[\begin{array}{lr}2 / 7 & 8 / 7 \\ 2 / 11 & -5 / 11\end{array}\right]$, i.e., (65), shown at the bottom of the next page. If $g_{i}=\tanh$ and $\int_{0}^{\infty} k_{i j}(s) d s=1$, then the trivial solution of system (65) is globally asymptotically stable [4], [10], [19]. 


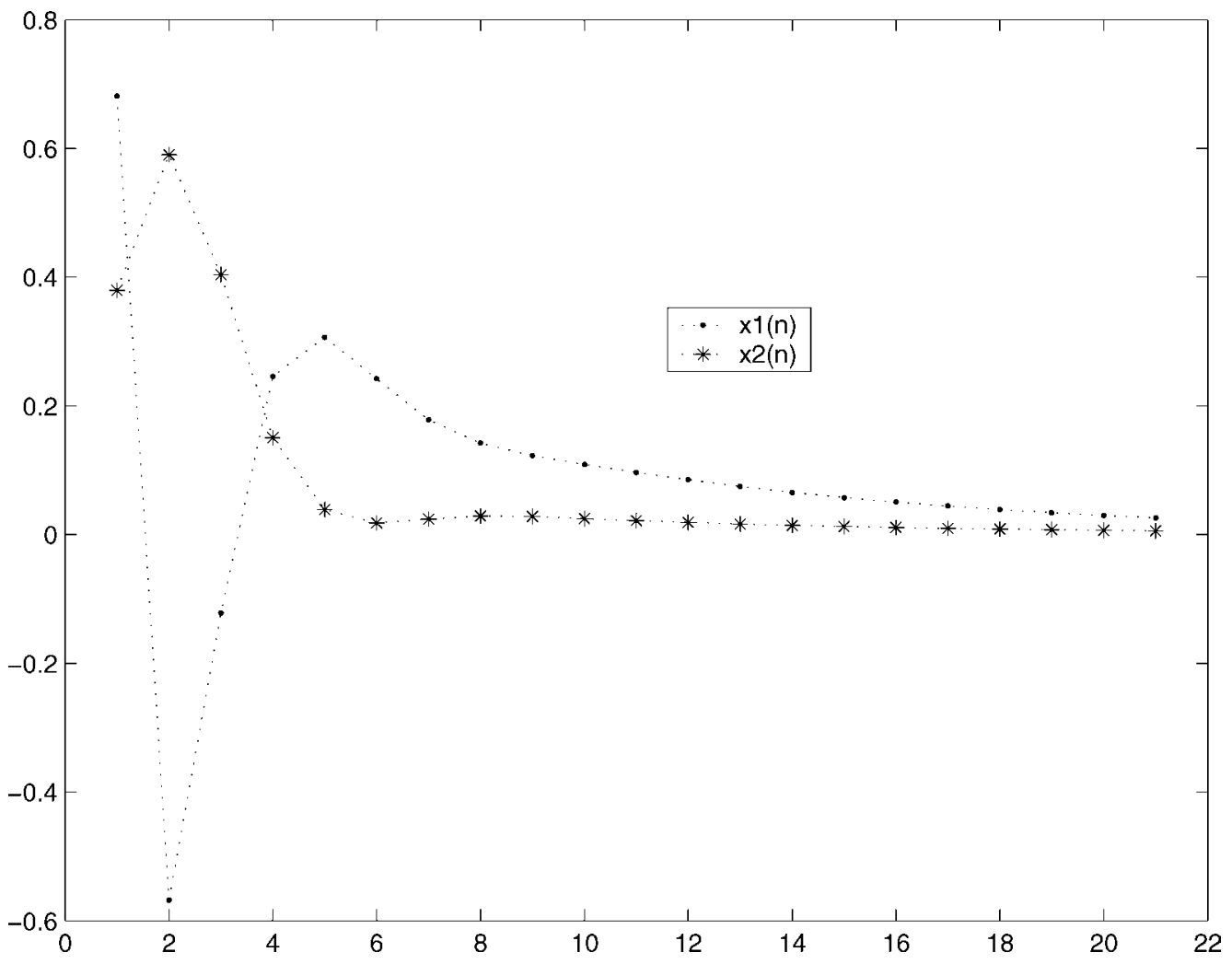

Fig. 2. Simulation of system (66) with $h=0.9 / 11$.

Using forward Euler approximation, we can write the discrete-time analog of system (65) as (66), shown at the bottom of the page. The stability of the trivial solution of system (66) definitely depends on the step-size of $h$. For computational purpose, we choose

$$
k_{11}[v]=k_{22}[v]=\frac{e-1}{e} e^{v}, k_{12}[v]=k_{21}[v]=\frac{(e-1)^{2}}{e} v e^{v}
$$

where $k_{11}$ is called fading memory, $k_{12}$ is called eventually fading memory, and both are normalized with $\sum_{v=0}^{\infty} k_{i j}[v]=1$. Fig. 2 shows the stability of the trivial solution with $h=0.9 / 11$ while Fig. 3 shows that when $h=1.05 / 11$, system (66) is unstable.

We now employ the unconventional finite difference method to consider the case of the same $h=1.05 / 11$. The stability property will maintain if we use the analogs (62) and (63) (see
Fig. 4). In fact, the stability dynamics of system (62) is consistent with its continuous-time counterpart without size restriction imposed on the discretization step $h$.

\section{CONCLUDING REMARKS}

We have established local stability result for discrete-time recurrent neural networks by linearizing them to Volterra difference systems, and the results are extremely useful to the networks that have more than one equilibria. The sufficient conditions in Section IV about global stability also guarantee the uniqueness of the equilibrium and thus one can avoid the difficulty in proving the existence and uniqueness of the equilibrium, which forms the underlying basis for the ad hoc constructing of a special Lyapunov function or functional. This is also why we start at the beginning to set the equilibrium at the origin (this can

$$
\left\{\begin{array}{c}
\dot{x}_{1}(t)=-7 x_{1}(t)+2 \int_{-\infty}^{t} k_{11}(t-s) g_{1}(s) d s+8 \int_{-\infty}^{t} k_{12}(t-s) g_{2}(s) d s \\
\dot{x}_{2}(t)=-11 x_{2}(t)+2 \int_{-\infty}^{t} k_{21}(t-s) g_{1}(s) d s-5 \int_{-\infty}^{t} k_{22}(t-s) g_{2}(s) d s
\end{array}\right.
$$

$$
\left\{\begin{array}{l}
x_{1}[n+1]=(1-7 h) x_{1}[n]+2 h \sum_{v=0}^{\infty} k_{11}\left([ v ] \operatorname { t a n h } \left(\left(x_{1}[n-v]\right)+8 h \sum_{v=0}^{\infty} k_{12}[v] \tanh \left(\left(x_{2}[n-v]\right)\right.\right.\right. \\
x_{2}[n+1]=(1-11 h) x_{2}[n]+2 h \sum_{v=0}^{\infty} k_{21}[v] \tanh \left(\left(x_{1}[n-v]\right)-5 h \sum_{v=0}^{\infty} k_{22}[v] \tanh \left(\left(x_{2}[n-v]\right)\right.\right.
\end{array}\right.
$$




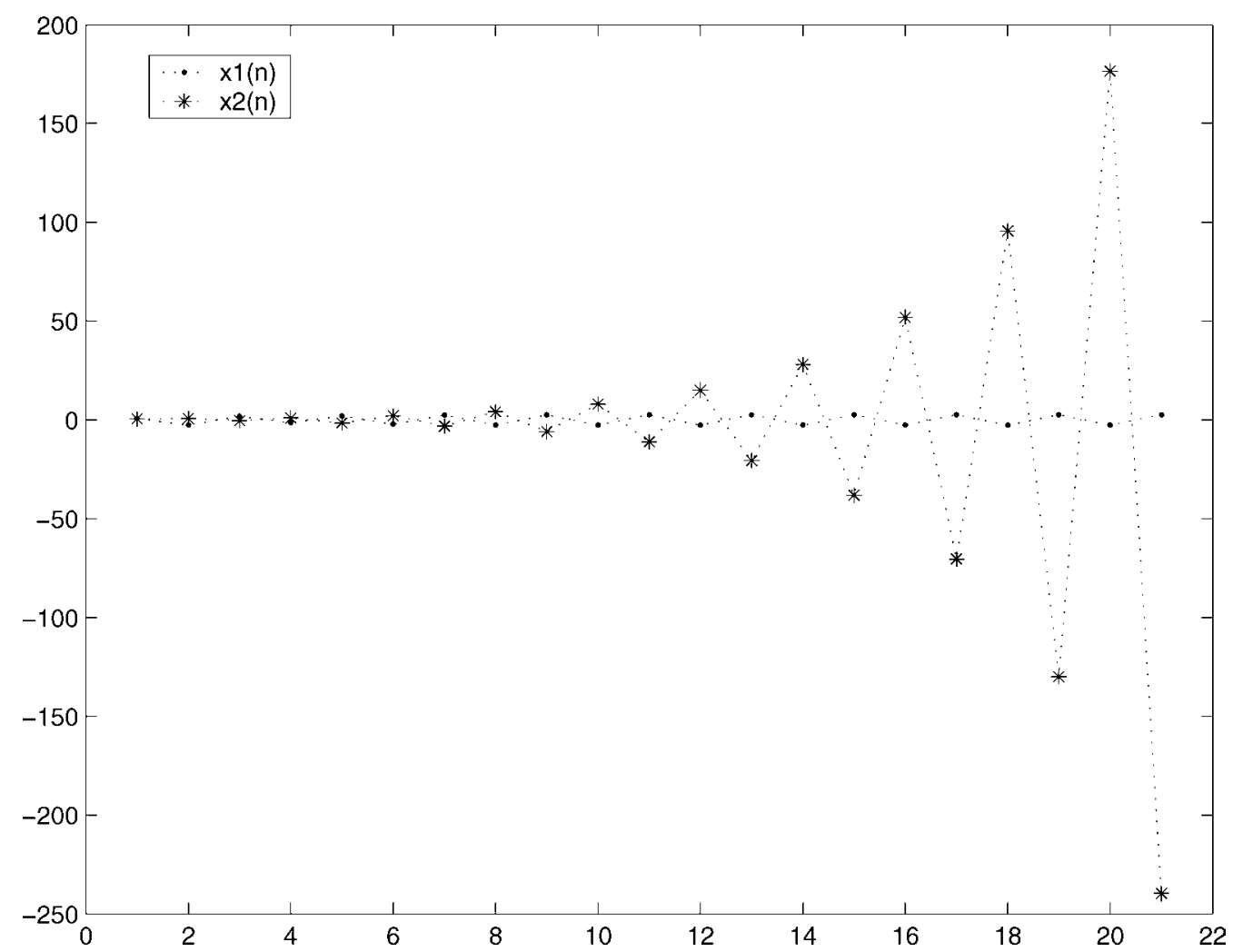

Fig. 3. Simulation of system (66) with $h=1.05 / 11$.

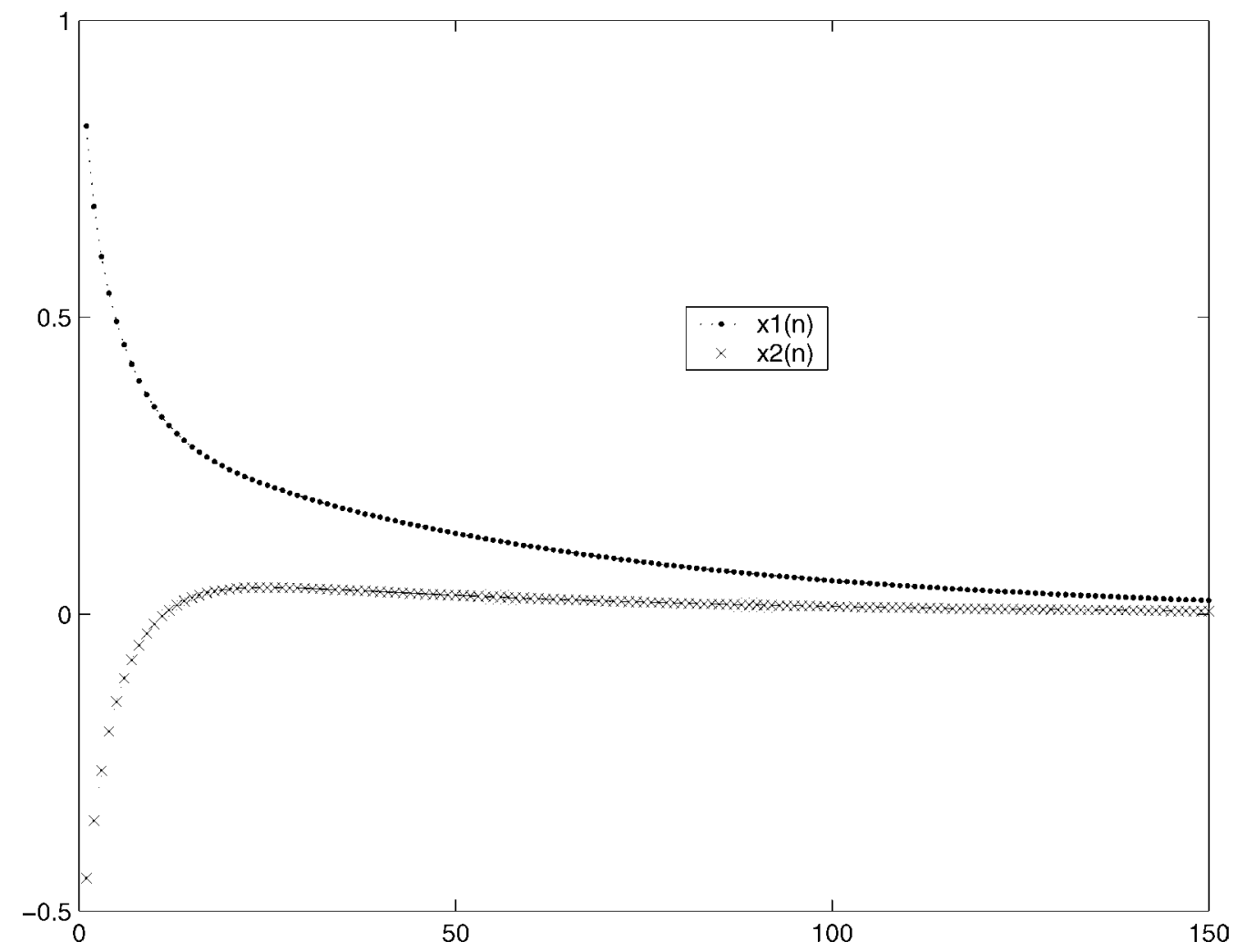

Fig. 4. Simulation of the discreet-time analog of system (65) using (62) and (63) with $h=1.05 / 11$.

always be achieved from a simple transformation). The generality of systems (12) and (13) is that they include not only finite and infinite memories, but also nearly all possible forms of discrete-time neural networks, as one can choose any possible combination of kernels $k_{i j}$ and functions $g_{i}$, like what we have done in Example 5.1. 
It is a trick and difficult task to discretize continuous-time neural networks, especially for computer simulation for delay systems, because their dynamical properties are not always consistent. From the unconventional finite difference method we have provided in the paper, we can see that one has to decrease the step-size or to strengthen the self regulation of the neural networks (through $\Phi(h)$ ) in order to guarantee the stability properties are heritable.

It is well known that the dynamics of continuous-time Hopfield neural networks with symmetric synaptic interactions is always convergent and the dynamics of their discrete-time analogs either convergent or approach to a two-periodic orbit. However, for a general discrete-time neural network, we can see from Example 5.1 in Section $V$ that its attractor may be very complex. Lyapunov exponent analysis can be used to determine the dimension of the attractor. If the attractor has noninteger dimension, then it is a stranger attractor or it exhibits chaos. To establish the structure of the attractor of a neural network, which usually has more than one equilibriums, will be a difficult but more challenging task.

\section{REFERENCES}

[1] S. Arik, "Global asymptotic stability analysis of bidirectional associative memory neural networks with time delays," IEEE Trans. Neural Netw., vol. 16, no. 3, pp. 580-586, May 2005.

[2] A. Berman and R. J. Plemmons, Nonnegative Matrices in the Mathematical Science. New York: Academic, 1977.

[3] E. K. Blum and X. Wang, "Stability of fixed points and periodic orbits and bifurcations in analog neural networks," Neural Netw., vol. 5, no. 4, pp. 577-587, Jul.-Aug. 1992.

[4] J. Cao and J. Wang, "Global asymptotic stability of a general class of recurrent neural networks with time-varying delays," IEEE Trans. Circuits Syst. I, Fundam. Theory Appl., vol. 50, no. 1, pp. 34-44, Jan. 2003.

[5] Y. Chen, "Global stability of neural networks with distributed delays," Neural Netw., vol. 15, no. 7, pp. 867-871, Sep. 2002.

[6] C. Feng and R. Plamondon, "On the stability analysis of delayed neural networks system," Neural Netw., vol. 14, no. 9, pp. 1181-1188, Nov. 2001.

[7] E. Goles-Chacc, F. Fogelman-Soulie, and D. Pellegrin, "Decreasing energy functions as a tool for studying threshold networks," Discrete Appl. Math., vol. 12, no. 3, pp. 261-277, Nov. 1985.

[8] S. Haykin, Neural Networks - A Comprehensive Foundations. New York: Macmillan, 1994.

[9] J. J. Hopfield, "Neural network and physical systems with emergent collective computational abilities," in Proc. Nat. Acad. Sci., 1982, vol. 79 , pp. $2554-2558$.

[10] S. Hu and J. Wang, "Global stability of a class of discrete-time recurrent neural networks," IEEE Trans. Circuits Syst. I, Fundam. Theory Appl., vol. 49, no. 8, pp. 1104-1117, Aug. 2002.

[11] X. Liu and J. Wang, "Solving pseudomonotone variational inequalities and pseudoconvex optimization problems using the projection neural network," IEEE Trans. Neural Netw., vol. 17, no. 6, pp. 1487-1499, Nov. 2006.

[12] L. Jin, P. N. Nikiforuk, and M. M. Gupta, "Absolute stability conditions for discrete-time recurrent neural networks," IEEE Trans. Neural Netw., vol. 5, no. 6, pp. 954-964, Nov. 1994.

[13] L. Jin and M. M. Gupta, "Global asymptotical stability of discrete-time analog neural networks," IEEE Trans. Neural Netw., vol. 7, no. 4, pp. 1025-1031, Jul. 1996.

[14] P. Koiran, "Dynamics of discrete time, continuous state Hopfield networks," Neural Comput., vol. 6, pp. 459-468, 1994.

[15] F. L. Lewis, Neural Network Control of Robot Manipulation and Nonlinear Systems. London, U.K.: Taylor \& Francis, 1999.

[16] X. Liao and J. Wang, "Global dissipativity of continuous-time recurrent neural networks with time delay," Phys. Rev. E, Stat. Phys. Plasmas Fluids Relat. Interdiscip. Top., vol. 68, p. 016118, Jul. 2003.

[17] P. Liu, "Stability of nonlinear discrete systems with applications to population dynamics," Ph.D. dissertation, Dept. Math. Statist., Flinders Univ., Adelaide, Australia, 1998.

[18] P. Liu and K. Gopalsamy, "Global stability and chaos in a population model with piecewise constant arguments," Appl. Math. Comput., vol. 101, no. 1, pp. 63-88, Jun. 1999.
[19] P. Liu and Q.-L. Han, "On stability of recurrent neural networks-An approach from Volterra integro-differential equations," IEEE Trans. Neural Netw., vol. 17, no. 1, pp. 264-267, Jan. 2006.

[20] S. Liu and J. Wang, "A simplified dual neural network for quadratic programming with its KWTA application," IEEE Trans. Neural Netw., vol. 17, no. 6, pp. 1500-1510, Nov. 2006.

[21] B. Müller, J. Reinhardt, and M. T. Dtrickland, Neural Networks- An Introduction, 2nd ed. New York: Springer-Verlag, 1995.

[22] S. Mohamad and K. Gopalsamy, "Exponential stability of continuoustime and discrete-time cellular neural networks," Appl. Math. Comput., vol. 135, no. 1, pp. 17-38, Feb. 2003.

[23] Y. Yang and J. Cao, "Solving quadratic programming problems by delayed projection neural network," IEEE Trans. Neural Netw., vol. 17, no. 6, pp. 1630-1634, Nov. 2006.

[24] Y. Xia, "An extended projection neural network for constrained optimization," Neural Comput., vol. 16, no. 4, pp. 863-883, Apr. 2004.

[25] _ _ "Further results on global convergence and stability of globally projected dynamical systems," J. Optim. Theory Appl., vol. 122, no. 3 , pp. 627-649, Sep. 2004.

[26] Y. Xia, G. Feng, and J. Wang, "A recurrent neural network with exponential convergence for solving convex quadratic program and related linear piecewise equations," Neural Netw., vol. 17, no. 7, pp. 1003-1015, Sep. 2004.

[27] Y. Xia and J. Wang, "A general projection neural network for solving monotone variational inequalities and related optimization problems," IEEE Trans. Neural Netw., vol. 15, no. 2, pp. 318-328, Mar. 2004.

[28] — - "A recurrent neural network for solving nonlinear convex programs subject to linear constraints," IEEE Trans. Neural Netw., vol. 16, no. 2, pp. 379-386, Mar. 2005.

[29] Z. Zeng and J. Wang, "Improved conditions for global exponential stability of recurrent neural networks with time-varying delays," IEEE Trans. Neural Netw., vol. 17, no. 3, pp. 623-635, May 2006.

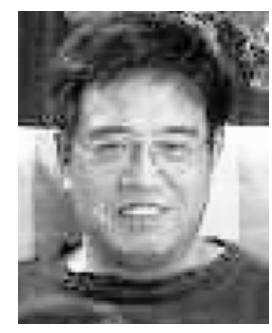

Pingzhou Liu received the B.Sc. degree in mathematics from the Shanxi Normal University, Linfen, China, in 1982, the M.Sc. degree in mathematics from Shaaxi Normal University, Xi'an, China, in 1987, and the Ph.D. degree in applied mathematics from the Flinders University, Adelaide, Australia, in 1998.

He was a Professor at the Shanxi Normal University and Research Associate/Lecturer at Flinders University. Currently, he is with the School of Computing Sciences, Faculty of Business and Informatics, Central Queensland University, Rockhampton, Australia, and also with the University of Adelaide. His research interests include dynamical systems and their applications, numerical analysis, and statistical reasoning.

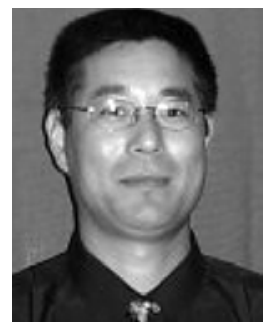

Qing-Long Han received the B.Sc. degree in mathematics from the Shandong Normal University, Jinan, China, in 1983 and the M.Eng. and Ph.D. degrees in information science (electrical engineering) from the East China University of Science and Technology, Shanghai, China, in 1992 and 1997, respectively.

From September 1997 to December 1998, he was a Post-Doctoral Researcher Fellow at LAII-ESIP, Université de Poitiers, Poitiers, France. From January 1999 to August 2001, he was a Research Assistant Professor in the Department of Mechanical and Industrial Engineering, Southern Illinois University, Edwardsville. In September 2001, he joined the Central Queensland University, Rockhampton, Australia, where he is currently an Associate Professor. He has held a Visiting Professor position in LAII-ESIP, Université de Poitiers, a Chair Professor position in Hangzhou Dianzi University, China, as well as a Guest Professor position in three Chinese universities. He has published over 140 refereed papers in technical journals and conference proceedings. His research interests include time-delay systems, robust control, networked control systems, neural networks, complex systems, and software development processes.

Prof. Han has been an Associate Editor for Dynamics of Continuous, Discrete \& Impulsive Systems - Series B: Applications \& Algorithms since 2004 and a Guest Editor for the Special Issue on Time-Delay Systems in that journal since 2006. From 2004 to 2006, he was a Guest Editor for the Special Issue on TimeDelay Systems in Asian Journal of Control. 